\title{
Study on the Optimum Technological Conditions of Germination of Buckwheat Seeds
}

\author{
Zhang Guotao \\ Yulin College, Yulin City 719000, Shaanxi Province, China \\ 994527145@qq.com
}

\begin{abstract}
Keywords: buckwheat, soak, germination
Abstract. To determine the optimum technological conditions of germination of buckwheat seeds, we studied on the influencing factors of germination rate of buckwheat seeds, which include soaking time, soaking temperature, germination temperature and germination humidity of buckwheat seeds. The results showed that the optimum germination yields of buckwheat seeds appeared at $30^{\circ} \mathrm{C}$ ambient temperature, $80 \%$ ambient humidity, and soaking seeds 10 hours at $30^{\circ} \mathrm{C}$.
\end{abstract}

\section{Introduction}

Buckwheat contains plenty of nutrients including proteins, vitamins, flavanoid, minerals and so on ${ }^{[1]}$. The health benefits of buckwheat such as antioxidation, anti-hypertension, anti-cancer, anti-diabetic, and cholesterol-lowering, are attributed to the unique composition of the buckwheat ${ }^{[2]}$. In the bodies the excess free radical can lead to biological aging and illness ${ }^{[3]}$. The buckwheat has materials with scavenging free radical, and more helpful composition for our body than common foods ${ }^{[4]}$. The more materials with scavenging free radical can be generated during the buckwheat seed germination ${ }^{[5]}$. In order to determining the changes of antioxidantive matters during the buckwheat seed germination, we study on the optimum technological conditions of the buckwheat seed germination in this paper.

\section{Materials and methods}

\subsection{Materials and equipment}

The buckwheat were bought from the local market. The constant temperature and humidity incubator was bought from Changzhou Zhongjie experimental instrument manufacturing company.

\subsection{Germination process}

Selection of buckwheat seeds $\rightarrow$ Cleaning of buckwheat seeds $\rightarrow$ Soaking of buckwheat seeds $\rightarrow$ Germination of buckwheat seeds $\rightarrow$ Collect buckwheat seeds germinating regularly

\subsection{Moisture absorption rate and Germination yields}

Moisture absorption rate $=\left(\mathrm{W}^{\prime}-\mathrm{W}\right) / \mathrm{W}$ (1); Germination yields $=\left(\mathrm{N}-\mathrm{N}^{\prime}\right) / \mathrm{N}$ (2) W': The weight of buckwheat seeds after soaking; W: The weight of buckwheat seeds; a: Soaking time. N: The number of buckwheat seed before germination; N': The number of buckwheat seed after germination.

\section{Results and discussion}

\subsection{The influencing factors of moisture absorption rate of buckwheat seeds}

Water is important to germination of buckwheat seeds. Water absorption is the first stage of germination of buckwheat seeds. The Fig. 1 showed the relationship between soaking time and moisture absorption rate of buckwheat seeds in different temperature. With soaking temperature increasing, the moisture absorption rate of buckwheat seeds was obviously improved. Moisture 
absorption of buckwheat seeds ran at the highest rate before 5 hours in different temperature, followed by higher rate from 5 hour to 10 hour. Although, water absorption of buckwheat seeds continued after 10 hour, this trends decreased significantly. If buckwheat seeds were soaked for too long time in water, they may become bad. But it was not enough for buckwheat seed germination for too little time.
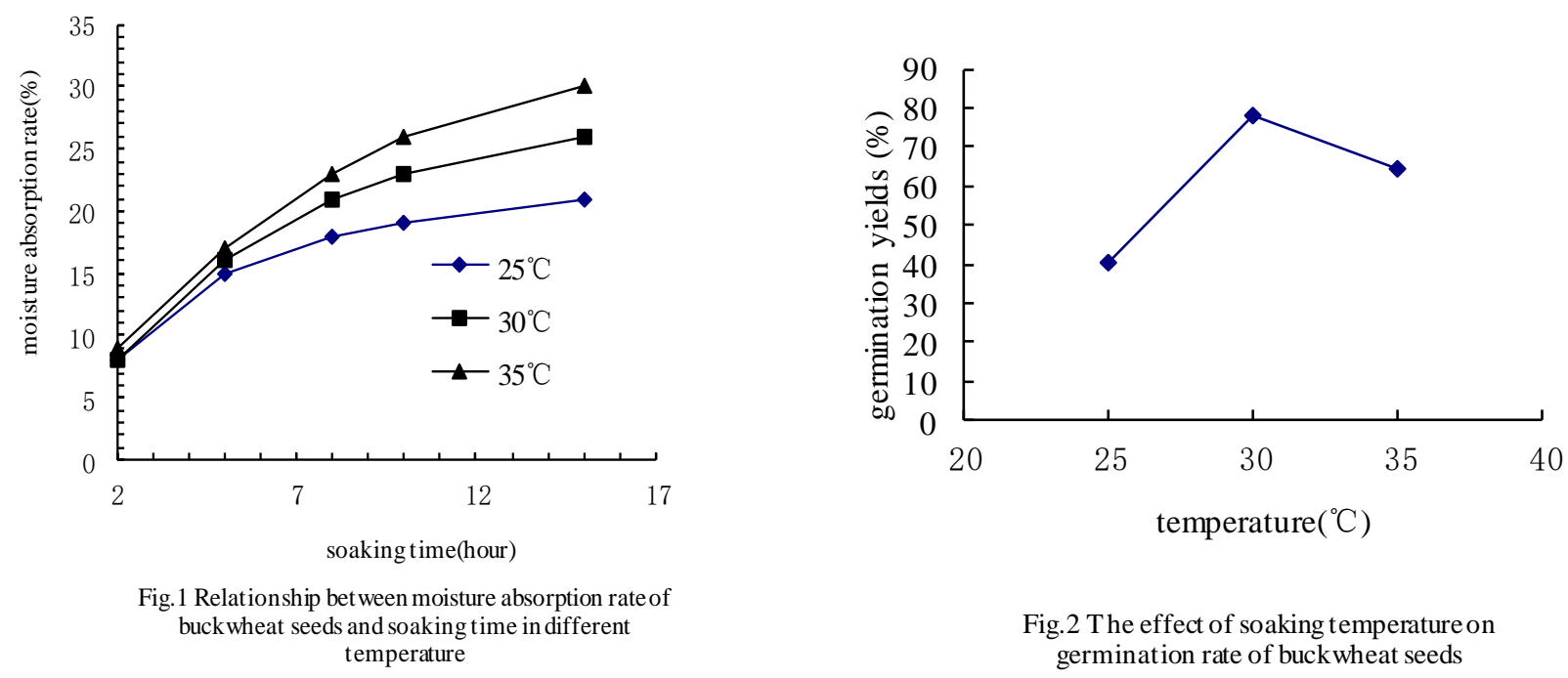

Fig.2 The effect of soaking temperature on germination rate of buck wheat seeds

\subsection{The effect of soaking temperature on germination yields of buckwheat seeds}

It was showed that buckwheat can germinate in different soaking temperature in Fig. 2, but there are different germination yields in different temperature. The highest germination yields appeared at $30^{\circ} \mathrm{C}$, followed by $35^{\circ} \mathrm{C}$, and finally at $25^{\circ} \mathrm{C}$. Therefor, the best soaking temperature is $30^{\circ} \mathrm{C}$.

\subsection{The effect of soaking time on germination yields of buckwheat seeds}

It was showed that germination yields of buckwheat seeds was measured at different soaking time in Fig.3. The maximum germination yields occured at 10 hour, followed by 15 hour, and finally at 5 hour. Therefore, the optimum soaking time is 10 hour.

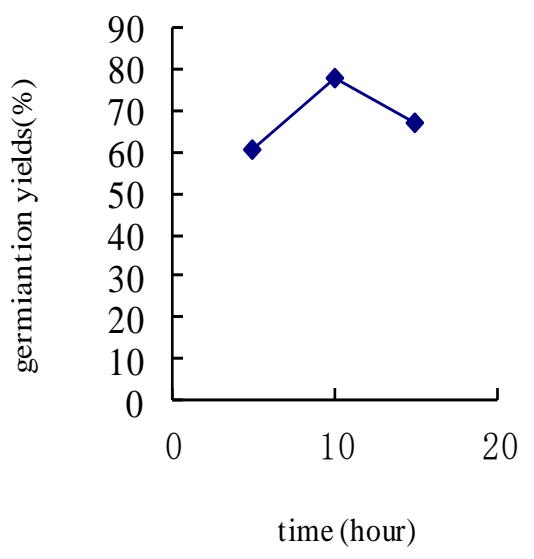

Fig.3 The effect of soaking timeon germination rate of buckwheat seeds

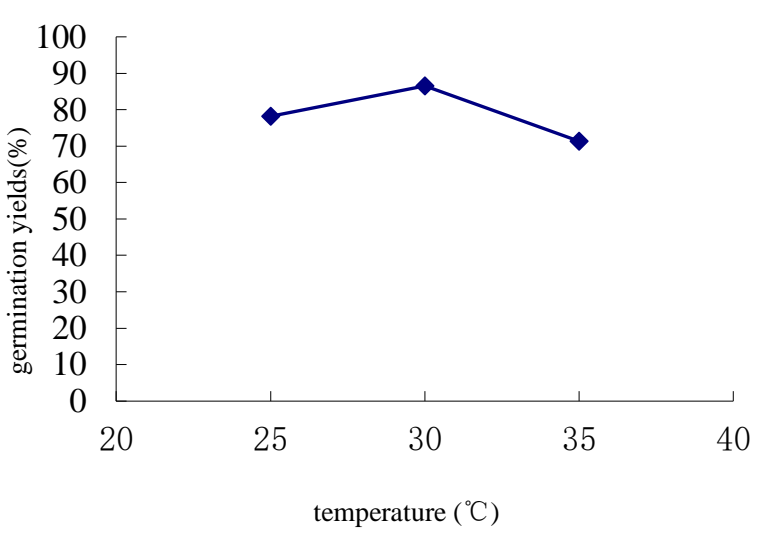

Fig.4 The effect of germination temperature on germination rate of buckwheat seeds 


\subsection{The effect of germination temperature on germination yields of buckwheat seeds}

Fig.4 showed that there are different germination yields of buckwheat seeds in different ambient temperature. With ambient temperature increasing, germination yields firstly increased, and then decreased. The optimum ambient temperature was $30^{\circ} \mathrm{C}$.

\subsection{The effect of germination humidity on germination yields of buckwheat seeds}

Germination of buckwheat seeds needs appropriate ambient humidity. The change of germination yields of buckwheat seeds was showed in Fig.5, with ambient humidity changing. To germination yields of buckwheat seeds, the optimum ambient humidity was $80 \%$, followed by $90 \%$, the finally $70 \%$.

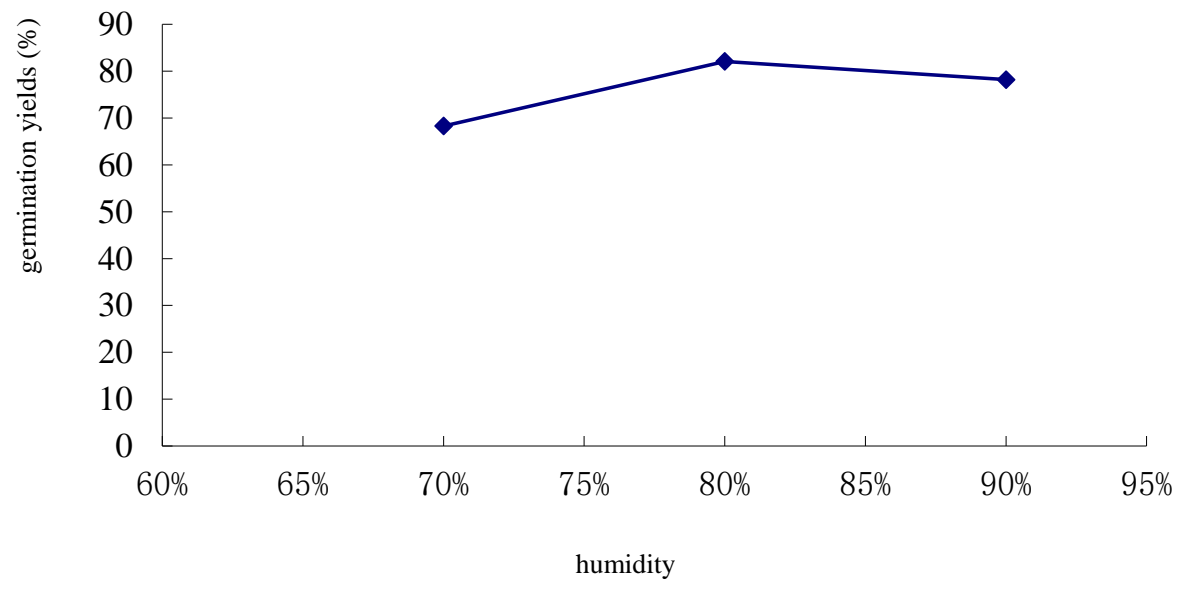

Fig.5 The effect of germination humidity on germination yields of buckwheat seeds

\section{Summary}

The influencing factors of buckwheat seeds germination included soaking temperature, soaking time, germination temperature and germination humidity. Our results demonstrated that buckwheat seeds had the optimum germination yields, when buckwheat seeds were soaked at $30^{\circ} \mathrm{C}$ for 10 hours. In addition, it was favorable for germination of buckwheat seeds that ambient temperature and ambient humidity were $30^{\circ} \mathrm{C}$ and $80 \%$ respectively.

\section{Acknowledgements}

This work was supported by the project of science and technology of Shaanxi Province (No. $2011 \mathrm{KW}-27$ ).

\section{References}

[1] F Zhu: Food chemistry Vol. 203 (2016), p. 231-245.

[2] L. S. Lee, E. J Choi, C. H. Kim, et al: Journal of cereal science Vol. 68(2016), p. 181-186.

[3] Pham-Huy LA, He H, Pham-Huy C, et al: International of journal of biomedical science Vol.4 (2008), p.89-96.

[4] G. Zhao, L. Peng, S. Wang, et al: Journal of integrative agriculture Vol.111 (2012), p. 1111-1118. 
[5] X. Zhou, T. Hao, Y. Zhou, et a: Journal food science technology Vol.52(2015), p. 2458-2463. 